Ann. Biol. anim. Bioch. Biophys., 1979, 19 (4 A), 1131-1140.

\title{
The effects of Isopropyl-N-Phenylcarbamate on meiotic maturation of mammalian oocytes
}

\author{
par Nicole CROZET, D. SZÖLLÖSI
}

Station centrale de Physiologie animale, I.N.R.A., 78350 Jouy en josas.

Summary. The effects of Isopropyl-N-Phenylcarbamate (IPC) on in vitro meiotic maturation were studied on mouse and rabbit oocytes. This drug, which has antimitotic properties on cells lacking centrioles (plants, algae, unicellular organisms), inhibits the oocyte meiotic maturation.

It prevents germinal vesicle breakdown and formation of cytoplasmic microtubules near the nucleus, as long as the oocytes are cultured in its presence. This effect is reversible in vitro ; however when IPC is applied before or at the onset of oocyte maturation, it induces disturbances in the orientation of forming microtubules of the Metaphase I-spindle.

In the mouse oocyte, IPC also causes an increase in number of nuclear bodies and their appearent continuity with other nuclear structures (condensed chromatin, nucleolus, granules).

Hypothesis is forwarded that these nuclear bodies would be the morphological expression of an accumulation of macromolecules (perhaps RNP in nafure) which would be stocked in the nucleus as long as the maturation process is not initiated. IPC acting on oocyte metabolism may either permit an accumulation of macromolecules or it may initiate a new wave of synthetic activity.

\section{Introduction.}

Isopropyl-N-Phenylcarbamate (IPC) is an herbicide which acts as an antimitotic agent. It apparently has a specific site of action effecting the spindle poles of plant cells which lack centrioles (Hepler and Jackson, 1969 ; Nasta and Gunther, 1973 ; Hervas, 1974). In contrast to the usual antimitotic agents eg. colchicine and its derivatives and vinblastine, this carbamate does not bind to tubulin (Coss et al., 1975) nor does it act on assembled microtubules; it seems to have an effect on the assembly of spindle microtubules directly at their site of polymerisation, at the microtubule organising centers (MTOCs) (Pickett-Heaps, 1971). The antimitotic action of carbamates has aiso been demonstrated on different algae (Coss and Pickett-Heaps, 1974) and unicellular organisms (Mergulis and Banerjee, 1969]; Brown and Bouck, 1974) in which the spindle is intranuclear and the poles are represented by MTOCs and not by centrioles. It seems that IPC is ineffective on cells which possess centrioles. Thus far only few attempts have been made on the action of carbamates upon animal cells. In preliminary studies (Laporte and Szöllösi, unpublished data) it was found that IPC does not effect the mito- 
tic rate of mouse L. cells in culture. However Oliver, Krawiec and Berlin (1978) indicate that Isopropyl $\mathrm{N}$-(3-chlorophenyl) carbamate inhibits the growth of mouse fibroblasts and causes morphological changes of the treated cells.

In mammalian oocytes, meiotic maturation is characterized by germinal vesicle breakdown (GVBD), condensation of diffuse dictyate chromatin, formation of the first meiotic spindle, emission of the first polar body and arrest at metaphase II if the oocyte is not fertilized or activated (Donahue, 1968). The GVBD is dependent (Calarco, Donahue and Szöllösi, 1972) on the polymerisation of cytoplasmic microtubules which are initiated at particular sites, the MTOCs, scattered around the germinal vesicle (GV). The meiotic spindle of oocytes differs from the spindle of all somatic cells in that it lacks centrioles (Szöllösi, Calarco and Donahue, 1972). One can take advantage of this unique property of oocytes among animal cells to initiate research on the action of carbamates upon mechanisms of meiotic maturation. In case these compounds would act upon the maturation process they could be used as new anti fertility agents which may be employed more punctually than the currently practiced methods involving steroids.

\section{Material and methods.}

Oocytes were obtained by puncturing preovulatory follicles of dissected ovaries from 2-to 4-month-old female Swiss albino mice or 3-month-old female New Zeland White rabbit in estrus. Oocytes containing an intact $G V$ were harvested with a micropipette and washed in culture medium (Brinster, 1971) (Brinster's medium, Gibco, New York), containing or not $2.10^{-4} \mathrm{M}$ Isopropyl-N-Phenylcarbamate (Sigma Chemical Co, St Louis, USA). Cell culture was carried out in $200 \mu \mathrm{l}$ of medium, under parafilm or paraffin oil, at $37{ }^{\circ} \mathrm{C}$, in humidified atmosphere of 5 p. $100 \mathrm{CO}_{2}$ in air. After culture, oocytes were washed and immediatly observed with a Zeiss photomicroscope equiped with Nomarski differential-interference optics, or directly treated for electron microscopy. For this purpose, oocyles were fixed for $40 \mathrm{~min}$ in $2 \mathrm{p} .100$ glutaraldehyde and 1 p. 100 paraformaldehyde in $0,075 \mathrm{M}$ phosphate buffer, containing 0,1-0,5 p. 100 potassium-ferricyanide; then post-fixed for 60 min in 2 p. 100 osmium tetroxyde. Subsequently the oocytes were placed for several hours or overnight, in 0,5 p. 100 aqueous uranyl acetate, at $4^{\circ} \mathrm{C}$. They were then dehydrated through a graded ethanol series and embedded in Epon 812 (Luft, 1961). Thin sections were obtained with a diamond knife on a Reichert $\mathrm{OMU}_{3}$ microtome and were stained with uranyl acetate $(30 \mathrm{~min})$ and lead citrate $(10 \mathrm{~min})$ before examination in a Zeiss EM $10 \mathrm{~B}$ electron microscope.

PLATE la, b. - Photomicrographs of mouse oocytes obtoined with Nomorsky differential-interference optics. Oocytes cultured 5 hrs in Brinster's medium (a) have undergone GVBD. Oocytes cultured 5 hrs with IPC (b) possess an intact GV (arrow) and nucleolus. $\times 600$.

c. - An electron micrograph of a mouse oocyle cultured $9 \mathrm{hrs}$ with IPC. The nuclear envelop (ne) is intact. Two nucleoli $(n)$ are present. Dense granules are associated with condensed chromatin (ch) and nucleoli. nb, nuclear body with microspherules $(\mathrm{m}) . \times 14000$.

d. - A rabbit oocyte cultured 5 hrs with IPC, showing an intact nuclear envelop (ne), nucleolus ( $n$ ) and condensed chromatin (ch). $\times 21000$. 


\section{Results.}

When mouse or rabbit oocytes are released from antral follicles and placed into suitable culture conditions, they immediatly undergo meiotic maturation, without hormonal stimulus. The timing of nuclear progression, in vitro, is known to occur in the mouse (Donahue, 1968) as follows : germinal vesicle breakdown takes place within 1-5 hrs, Metaphase I within 4-9 hrs and Metaphase II within 11-17 hrs. In the rabbit the events occur slower : GVBD is about 4-6 hrs and the first polar body is emitted within 7-10 hrs (Chang, 1955 ; Thibault, 1972).

After 5 hrs of culture in Brinster's medium, we find actually that 86 p. 100 of mouse oocytes (PI. la) have undergone GVBD. In contrast, 76 p. 100 of oocytes cultured during the same time in presence of IPC $(\mathrm{PI} . \mid b)$ do not resume meiosis. They have intact GV and nucleolus(i).

At ultrastructural level, the mouse oocyte cultured with IPC (PI. Ic) presents an intact nuclear envelop along which pores are visible. The nucleus contains usually one or, sometimes two, spherical nucleoli. They are very electron-dense and compact structures composed only of fine filamentous elements. In the nucleoplasmi there are few areas of condensed chromatin, nuclear bodies (nb) and electron-dense granules about 50-60 $\mathrm{nm}$ in diameter usually associated with chromatin, or near the nucleolus. The $\mathrm{nb}$ are characterized by a fibrillar matrix (PI. Ila) including granules about 15-20 nm in diameter. Dense microspherules are often seen at their periphery; they are probably heterochromatic knobs as suggested by Chouinard (1973). These nuclear structures are similar to those observed in oocytes from antral-follicles, but the nb are more numerous (as shown by serial sections of the nucleus) and present more frequently continuity with other elements such as the nucleolus, granules and chromatin (PI. IIb). In the cytoplasm near the GV neither microtubuless nor MTOCs are seen.

In the rabbit we also observed that IPC inhibits oocyte GVBD. Oocyles cultured during $5 \mathrm{hrs}$ in Brinster's medium have undergone GVBD, while oocytes cultured in presence of the drug possess (PI. Id) an intact GV and a nucleolus. In the nucleoplasm there are patches of condensing chromatin as in control oocytes at the outset of the culture. Neither microtubules nor MTOCs are present in the cytoplasm.

When mouse oocytes are placed for $30 \mathrm{~min}$ in medium free of IPC, before continuation of culture with IPC, nuclear progression is inhibited (PI. IIIa); they are similar ultrastructurally to oocytes directly cultured in presence of the drug. But the arrest of maturation process appears to be reversible. Effectively, if these oocytes are cultured for the duration of about $3 \mathrm{hrs}$ in a drug-free medium following $3 \mathrm{hrs}$ in presence of IPC, the GVBD takes place (PI. IIlb) ; the chromosomes condense (PI. IIIc) there are microtubules and MTOCs (PI. IIlc, d). In few cases a nuclear body was still present

PLATE Ila, b. - Mouse oocytes cultured 9 hrs with IPC. Detail of nuclear body. The fibrillar matrix (arrows) include small granules. Microspherules $(\mathrm{m})$ represent probably heterochromatic knobs (a). $\times 24000$.

A nuclear body ( $\mathrm{nb}$ ) showing continuity with granules (arrows); chromatin (ch); microspherule (m) (b). $\times 40000$. 


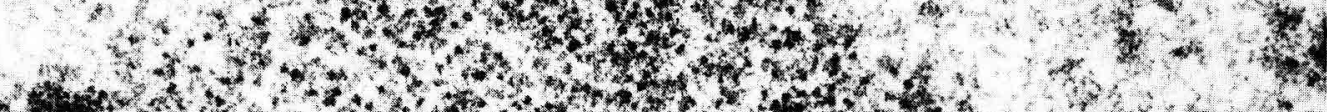

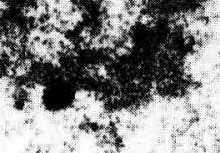

(4)

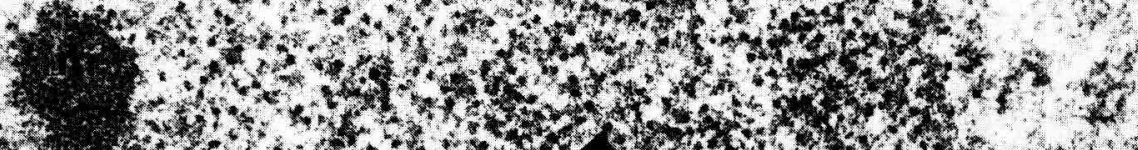

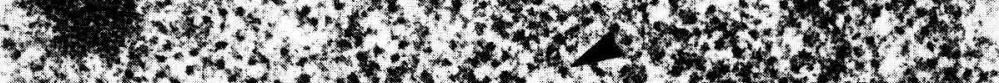

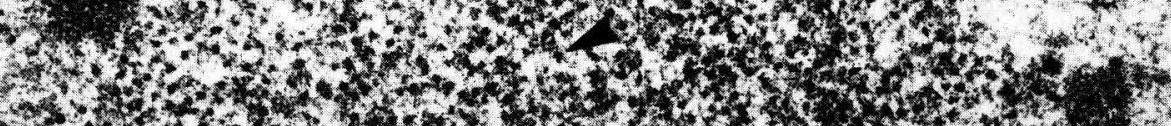

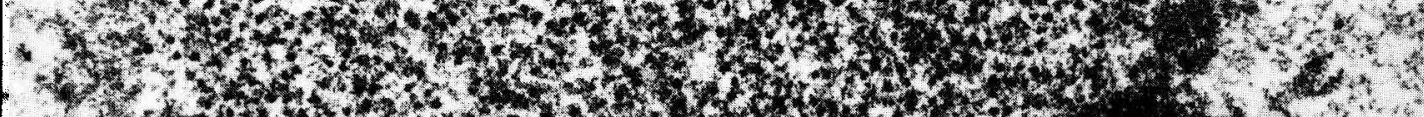
- S 3 .

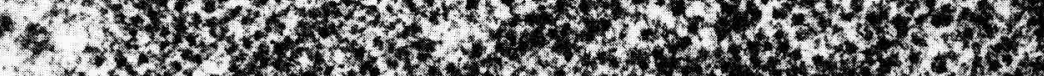

20
$y$

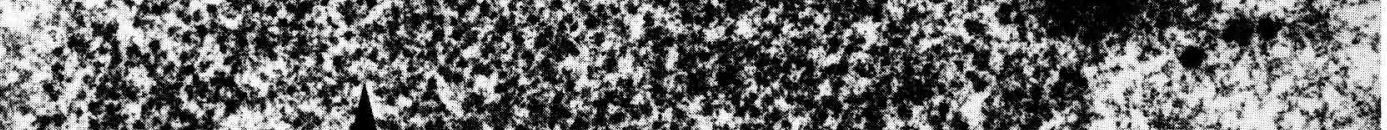

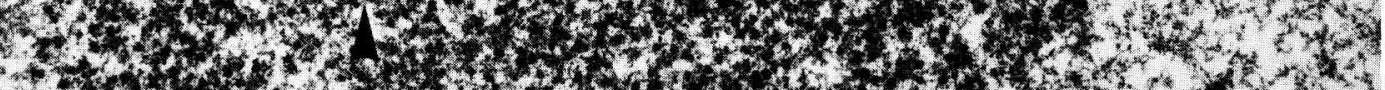

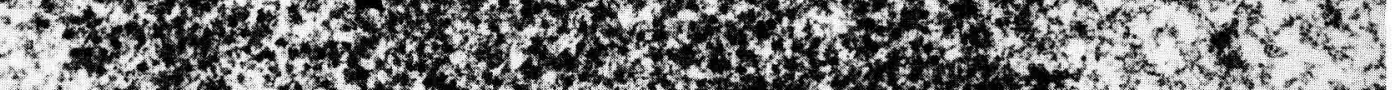

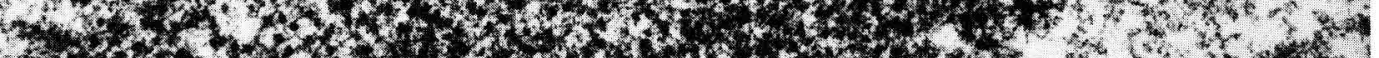

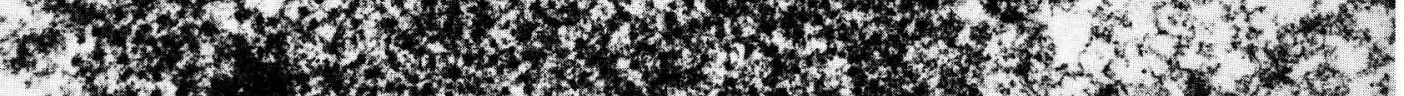

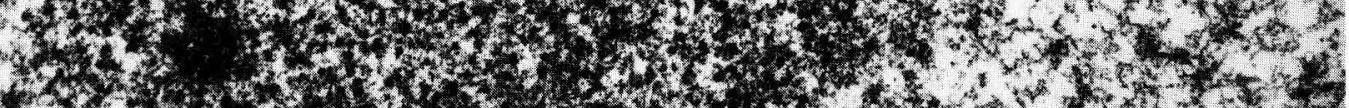

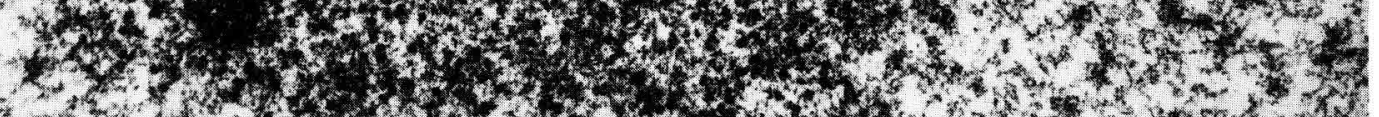

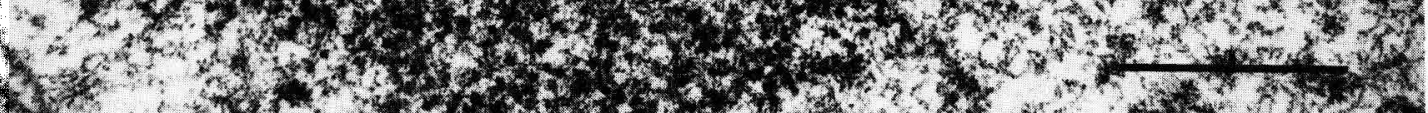

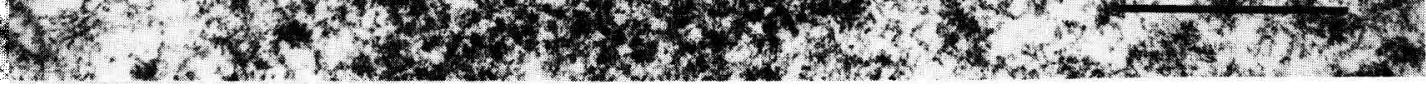

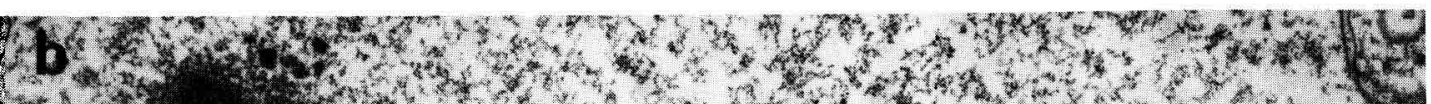

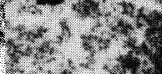

$a^{3}{ }^{2}:-3.38$

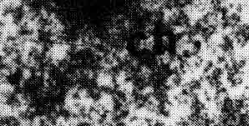

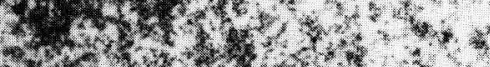

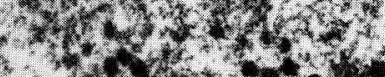

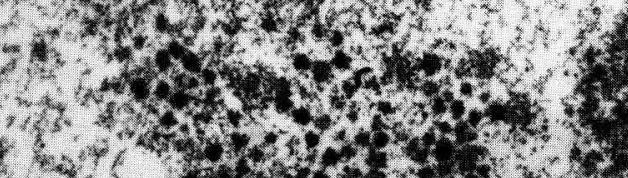

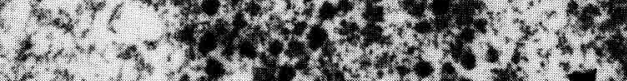

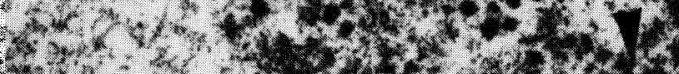

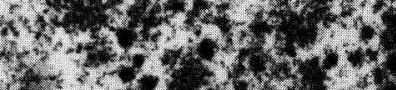

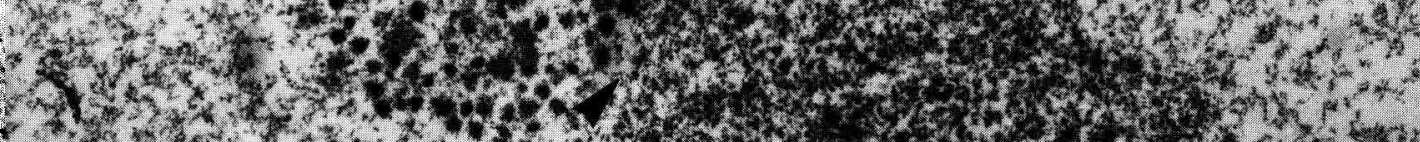

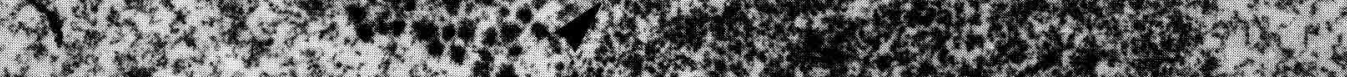

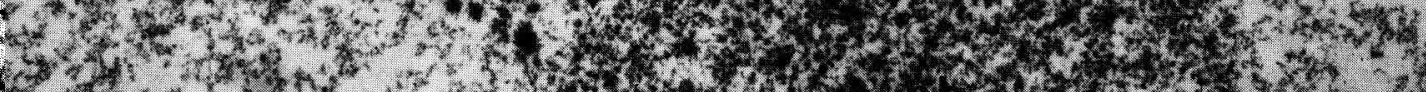

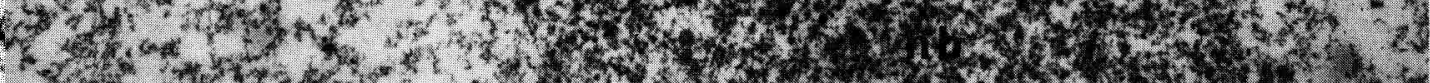
s.

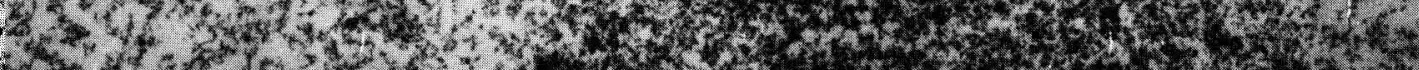

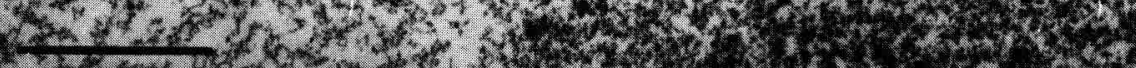


(PI. IIId). In such specimen the microtubules appear randomly oriented between chromosomes and the MTOCs (about 3 on the same section) are distributed around the chromosomes and not at two poles. One or two chromosomes appears to be lagging outside the spindle. These observations indicate a disturbance in spindle formation.

The effect of IPC after occurence of GVBD was studied on mouse oocytes cultured during 3 hrs Brinster's medium followed by a 3 hrs of culture with IPC (PI. IVb). In these cases we noticed little changes on the microtubules orientation and spindle organization compared with normally matured oocytes ( $\mathrm{Pl}$. IVa). Still sometimes a chromosome remains outside of the spindle.

\section{Discussion.}

IPC acts in two different ways on oocyte meiotic maturation : the GVBD and spindle formation. The failure of the GVBD occurs in 76 p. 100 of mouse oocytes which represent important rate compared to the 86 p. 100 of oocytes which resume meiosis in vitro under normal conditions. The oocyles do not mature as long as they are in presence of IPC, even if they were placed previously in Brinster's medium for $30 \mathrm{~min}$, a medium which is able to initiate nuclear progression if cultured for longer period. But this inhibitory effect of IPC on GVBD appears to be reversible. The disorientation of the spindle microtubules occurs when oocytes are cultured with IPC before or at the first sign of maturation. This may indicate that this drug affects spindle formation ; when oocytes were cultured in drug-free medium after IPC treatment a more extensive spindle disturbance is noted. In contrast, it seems that IPC has no effect on the orientation of preexisting spindle-microtubules. In the rare occasion, when IPC treatment following $3 \mathrm{hrs}$ of culture in normal medium, a lagging chromosome or slight spindle desorganization was found, may be due to the fact that particular oocyte was delayed and microtubule polymerisation was in process when the drug was applied.

These results correspond with those obtained on other cells lacking centrioles : algae (Coss and Pickett-Heaps, 1974) unicellular organisms (Mergulis, and Banerjee, 1969 ; Brown and Bouck, 1974), plant cells (Hepler and Jackson, 1969 ; Hervas, 1974) and tend to demonstrate that IPC reacts with MTOCs and not directly with microtubules ; but its mechanism of action remains still unknow.

A further effect of IPC thus far observed only in mouse oocytes is the increase in number of nuclear bodies and their appearent continuity with other nuclear structures. Chouinard $(1973,1975)$ has observed that nuclear bodies are present in relatively large numbers in the growing mouse oocytes, at the onset and during the stage

PLATE IIla. - Mouse oocytes placed 30 min in Brinster's medium, then cultured 6 hrs with IPC. Nuclear progression is inhibited (Nomarsky photomicrograph). $\times 200$.

b, c, d. - Mouse oocytes cultured 3 hrs in drug free-medium following 3 hrs with IPC. GVBD has occured. Nomarsky photomicrograph (b). $\times 200$.

Electron micrographs. Chromosomes (ch) are condensed, microtubules randomly oriented (arrows) and microtubule organizing centers (MTOC) are present. nb, residual nuclear body (c-d). $\times 40000$. 
of follicle development; during these periods they are associated with segments of the condensing bivalents. On the contrary, through the final stage of antral follicle development when oocyte growth is slight most nuclear bodies detach from their associated bivalents and undergo a gradual shrinkage. At the preovulatory follicle stage few nuclear bodies are present. This last point is consistent with our observations on the preovulatory oocytes used for the experiments. Nuclear bodies are numerous when the GV is not in a pre-breakdown stage similar to the IPC treated oocytes. We would expect that nuclear bodies represent morphological expression of an accumulation of macromolecules in the nucleus, where they would continue to be stocked as long as the maturation process is not initiated. IPC acting on oocyte metabolism may either permit an accumulation of macromolecules or it may initiate a new wave of synthetic activity. Nuclear bodies form and accumulate at the onset and during the stage of follicle development when the rate of RNA synthesis is elevated. Moreover the observations of Palombi and Viron (1977) based on EDTA regressive coloration of mouse oocytes on frozen sections indicate that these nuclear bodies could be RNP in nature. It seems probable that nuclear bodies, as suggested by Chouinard (1973) are in relation with genetic activity of the dictyate chromosomes. These nuclear bodies would be expressions of accumulation of RNP products. According to this hypothesis it remains to be determined if IPC may induce a reinitiation of synthesis of RNP products.

\section{Conclusion.}

The resulis reported here show that IPC is effective on the process of the meiotic maturation of mammalian oocytes. It prevents the GVBD and disturbs the orientation of spindle microtubules probably by interaction with the MTOCs. It seems also to modify the oocyte metabolism. The changes in number of the nuclear bodies and their continuity with other nuclear structures would be the expression of modifications occuring either in the production or stockage or processing of some RNA products.

Reçu en mars 1979.

Accepié en mors 1979.

Aknowledgements. - This work was financially supported in part by Ford Foundation Grant $n^{0} 770-0315$.

Résumé. L'action de I'Isopropyl-N-Phenylcarbamate (IPC) sur la maturation in vitro des ovocytes a été étudiée chez la Souris ef la Lapine. Cet agent, dont les propriétés antimitotiques vis-à-vis des cellules dépourvues de centrioles (plantes supérieures, algues, organismes unicellulaires) sont bien connues, apparaît susceptible de bloquer la maturation ovocytaire.

Il empêche l'éclatement de la vésicule germinative et la formation de microtubules cytoplasmiques autour du noyau tant que les ovocytes sont cultivés en sa présence. Cet effet

PLATE IVa. - Metaphose I of normally matured in vitro oocyle; microtubules (arrows). $\times 23000$.

b. - Mouse oocyte cultured 3 hrs in Brinster's medium then 3 hrs with IPC. Changes are not seen either in the microtubules orientation (arrows) or spindle organization. $\times 16000$. 


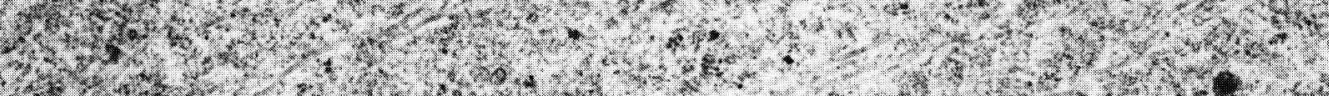

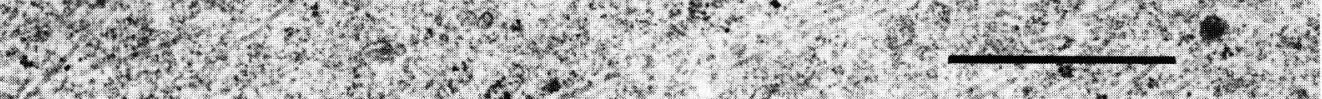

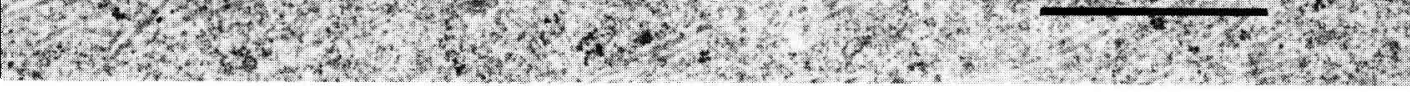

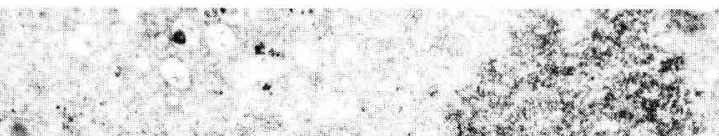

ef

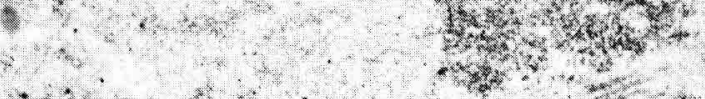

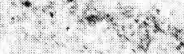

1.1. 37

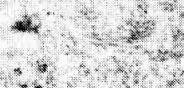

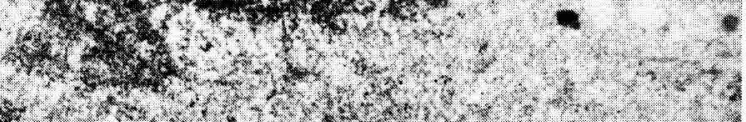

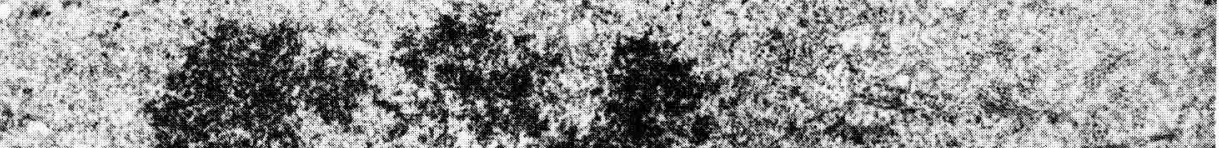

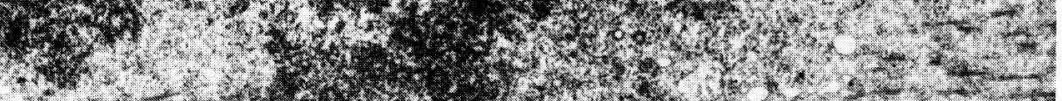

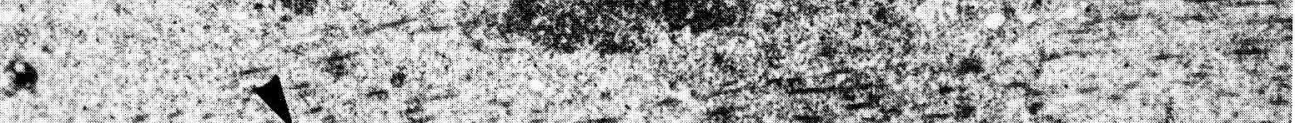

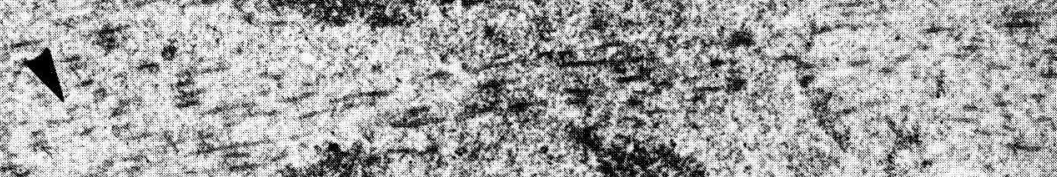

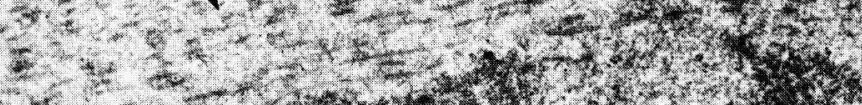

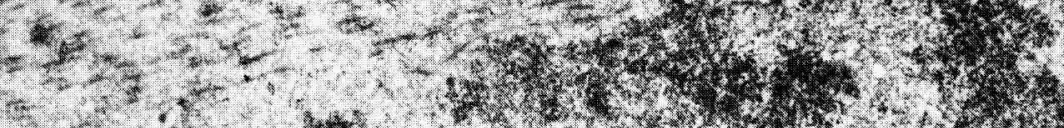

20

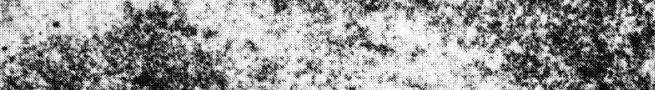


est réversible in vitro; cependant, lorsque I'IPC a réagit avec les ovocytes avant ou pendant le début de la maturation, il entraîne des perturbations dans l'orientation des microtubules du fuseau (Métaphase I) en cours de formation.

Chez la Souris, il induit, en outre, une augmentation du nombre des corps nucléaires qui présentent alors certaines continuités avec d'autres éléments du noyau (chromatine en cours de condensation, nucléole, granules). Une hypothèse esł émise, selon laquelle ces corps nucléaires seraient l'expression morphologique d'une accumulation de macromolécules (peut-être de nature RNP) qui resteraient stockées dans le noyau tant que les processus de maturation ne sont pas déclenchés. L'IPC agissant sur le métabolisme de l'ovocyte pourrait être à l'origine d'un nouveau stockage de macromolécules préexistantes ou néosynthétisées.

\section{References}

BRINSTER R. L., 1971. In vitro culture of the embryo. In Pathways to conception, Ch. G. Thomas Publ. Co., Springfield, III.

BROWN D. L., BOUCK G. B., 1974. Microtubule biogenesis and cell shape in Ochromonas. III. Effects of the herbicidal mitotic inhibitor Isopropyl-N-Phenylcarbamate on shape and flagellum regeneration. J. Cell Biol., 61, 514-536.

CALARCO R., DONAHUE R. P., SZÖLLÖSI D., 1972. Germinal vesicle breakdown in the mouse oocyte. J. Cell Sci., 10, 1-17.

CHANG M. C., 1955. The maturation of rabbit oocytes in culture and their maturation, activation, fertilization and subsequent development in the fallopian tubes. J. exp. Zool., 128, 379-405.

CHOUINARD L. A., 1973. An electron-microscope study of the extranucleolar bodies during growth of the oocyte in the prebubertal mouse. J. Cell Sci., 12, 55-70.

CHOUINARD L. A., 1975. A light- and electron-microscope study of the oocyte nucleus during development of the antral follicle in the prebubertal mouse. J. Cell Sci., 17, 589-615.

COSS R. A., PICKETT-HEAPS J. D., 1974. Effects of Isopropyl-N-Phenylcarbamate on the green alga Oedogonium Cardiacum. I. Cell division. J. Cell Biol., 63, 84-98.

COSS R. A., BLOODGOOD R. A., BROWER D. L., PICKETT-HEAPS J. D., MCINTOSH J. R., 1975. Studies on the mechanism of action of Isopropyl-N-Phenylcarbamate. Exp. Cell Res., 92, 394398.

DONAHUE R. P., 1968. Maturation of the mouse oocyte in vitro. I. Sequence and timing of nuclear progression. J. exp. Zool., 169, 237-250.

HEPLER P. K., JACKSON W. T., 1969. IsopropyI-N-Phenylcarbamate affects spindle microtubule orientation in dividing endosperm cells of Haemanthus Katherinae Baker. J. Cell Sci, , 5, 727-743.

HERVAS J. P., 1974. Antimitotic activity of Isopropyl-N-Phenylcarbamate in meristematic cells. Kinetics of the production of multipolar anaphasis. Arch. Biol., 85, 453-459.

LUFT J. H., 1961. Improvements in epoxy resin embedding methods. J. Biophys. Biochem. Cytol., 9, 409-414.

MERGULIS L., BANERJEE D., 1969. Effects of mitotic spindle inhibitors on regenerating cilia of Stentor Coeruleus. J. Protozool., 16, $7 \mathrm{~S}$.

NASTA A., GUNTHER E., 1973. Mitotic disturbances by application of carbamate herbicides on Allium cepa and Hordeum vulgare. Biol. Zentralbl., 92, 27-36.

OLIVER J. M., KRAWIEC J. A., BERLIN R. D., 1978. A carbamate herbicide causes microtubule and microfilament disruption and nuclear fragmentation in fibroblasts. Exp. Cell Res., 116, 229-237.

PALOMBI F., VIRON A., 1977. Nuclear cytochemistry of mouse oogenesis. I. Changes in extranucleolar ribonucleoprotein components through meiotic prophase. J. Ulitrastruct. Res., 61, 10 20.

PICKETT-HEAPS J. D., 1971. The autonomy of the centriole : fact or fallacy ? Cytobios, 3, 205-214.

SZÖLLOSI D., CALARCO P., DONAHUE R. P., 1972. Absence of centrioles in the first and second meiotic spindles of mouse oocytes. J. Cell Sci., 11, 521-541.

THIBAULT C., 1972. Final stages of mammalian oocyte maturation, 397-411. In BIGGERS J. D., SCHUETZ A. W., Oogenesis, Univ. Park Press, Baltimore. 